[Review]

\title{
用于生物电化学系统的石墨烯电极新进展
}

\author{
王 强 ${ }^{1}$ 黄丽萍 ${ }^{1, *} \quad$ 于洪涛 $^{1}$ 全 熟 ${ }^{1, *} \quad$ 陈国华 ${ }^{2}$ \\ ('大连理工大学环境科学与技术学院, 工业生态与环境工程教育部重点实验室, 辽宁 大连 116024; \\ 2香港科技大学化学与生物分子工程系, 香港九龙)
}

\begin{abstract}
摘要: 可持续社会的发展需要成本低, 并从废物或废水中提取能源或将能源转化为产品的环境友好技术. 近 年兴起的生物电化学系统(BESs)利用微生物催化不同电化学反应, 是将废物或废水中能量转化为电能等多种 产品的发展前景广阔的新技术. 当有关反应的吉布斯自由能小于零, 系统输出电能, 此时的 BESs 即为微生物 燃料电池(MFCs); 相反, 若反应的吉布斯自由能为正值, 此时的 BESs 被称为微生物电解电池(MECs). 随着研 究工作的不断深入和拓展, BESs 的电极性能已成为制约其应用的瓶颈. 石墨烯以其独特的结构和优异的材料 性能在 BESs 领域, 特别是 MFCs 中得以应用. 本文参考了最新的文献资料, 综述了石墨烯应用于BESs 的发展 现状, 包括应用于MFCs 的石墨烯电极、掺杂石墨烯电极、担载石墨烯电极, 对其在 MECs 中可能的应用, 以及 未来发展趋势予以展望.
\end{abstract}

关键词：石墨烯；电极；生物电化学系统；微生物燃料电池； 微生物电解电池 中图分类号: 0646

\section{Recent Developments of Graphene Electrodes in Bioelectrochemical Systems}

\author{
WANG Qiang ${ }^{1} \quad$ HUANG Li-Ping ${ }^{1, *} \quad$ YU Hong-Tao ${ }^{1} \quad$ QUAN Xie ${ }^{1, *} \quad$ CHEN Guo-Hua ${ }^{2}$
}

('Key Laboratory of Industrial Ecology and Environmental Engineering, Ministry of Education, School of Environmental Science and Technology, Dalian University of Technology, Dalian 116024, Liaoning Province, P. R. China; ${ }^{2}$ Department of Chemical and Biomolecular Engineering, Hong Kong University of Science and Technology, Kowloon, Hong Kong, P. R. China)

\begin{abstract}
Sustainable societies require development of cost-effective methods for harnessing energy from wastes and wastewater, and alternatively capturing this energy to make other useful chemicals with simultaneous wastes and wastewater treatment. Recently developed bioelectrochemical systems (BESs) that use microorganisms to catalyze different electrochemical reactions are promising for capturing the energy in wastes and wastewater for diverse purposes. A BES is called a microbial fuel cell (MFC) if electricity is generated and the Gibbs free energy change of the corresponding reaction is negative. Conversely, when the Gibbs free energy change of the overall reaction is positive, power needs to be supplied to drive this non-spontaneous reaction, and this BES is regarded as a microbial electrolysis cell (MEC). The electrode character is considered to be a key factor for triggering the applicable BESs. Graphene has been recently used as the electrode and investigated in BESs because of its unique structure and excellent properties. Here, an up-to-date review is provided on the recent research and development in BES-based graphene, particularly in MFC-based graphene. The recent pristine graphene,
\end{abstract}

Received: January 14, 2013; Revised: March 14, 2013; Published on Web: March 15, 2013.

"Corresponding authors. HUANG Li-Ping, Email: lipinghuang@dlut.edu.cn; Tel: +86-411-84708546. QUAN Xie, Email: quanxie@dlut.edu.cn; Tel: +86-411-84706140

The project was supported by the National Key Basic Research Program of China (973) (2011CB936002), Natonal Natural Science Foundation of China (51178077, 21077017), and Specialized Research Fund for the Doctoral Program of Higher Education, China (20120041110026).

国家重点基础研究发展规划项目(973) (2011CB936002), 国家自然科学基金(51178077, 21077017)及教育部高等学校博士学科点专项科研基金 (20120041110026)资助

(C) Editorial office of Acta Physico-Chimica Sinica 
doped graphene, and supported graphene research in MFCs is described in detail. The potential applications of graphene in MECs and the scientific and technical challenges are also discussed.

Key Words: Graphene; Electrode; Bioelectrochemical system; Microbial fuel cell; Microbial electrolysis cell

\section{1 引 言}

生物电化学系统(BESs) 是微生物燃料电池 (MFCs) 和微生物电解电池(MECs) 的统称, 具有清 洁、可持续等诸多优点, 在污染物的降解、污染场地 的修复、可植入式医学设备、生物传感器、海水淡 化、有价产品制备与清洁能源(氢气、电能)产出等方 面展示着广阔的应用前景, 引起人们的广泛关注. ${ }^{1-3}$ 随着研究工作的不断深入和拓展, BESs 电极性能, 特别是电极的较小比表面积、较大电阻已成为制约 其性能发挥和实际应用的瓶颈. 寻找高性能、低成 本的新型 BESs 电极材料是当前人们关注的热点. $2 ., 5$

与传统的 BESs 电极如碳布、碳纸、碳刷、碳毡、 碳纤维、石墨颗粒等不同, ${ }^{5}$ 石墨烯是近年发现的碳 材料的新成员, 它是由 $s p^{2}$ 杂化碳原子形成的二维蜂 窝状晶体, 具有载流子迁移率最高、高比表面积、良 好的电化学催化、较高的机械强度等奇特性质, 引 起了科学家的极大兴趣, 已应用于超级电容器、锂 离子电池、分解水、电催化燃料电池和染料敏化太 阳能电池等方面..$^{6-8}$ 石墨烯可分为石墨烯薄膜和功 能化石墨烯, 功能化石墨烯是通过化学修饰的方法 使石墨烯掺杂电负性原子 $(N 、 S 、 P 、 B)$ 或担载功能性 化合物和金属单质, 而功能化的石墨烯显现出更优 异的电化学性能, 在有机合成、传感器、环境保护和 能源系统中得到应用. 9

在 BESs 中, 电子由阳极输出经过外电路进入 阴极. 对于输出电子的阳极电极而言, 较大的比表 面积和良好的生物相容性, 有助于附着更多的电活 性微生物, 而材料较高的导电性有利于减小电极的 过电势, 从而减小电荷转移阻力. ${ }^{1}$ 研究表明, 石墨烯 与功能化的石墨烯均能催化 $\mathrm{K}_{3}\left[\mathrm{Fe}(\mathrm{CN})_{6}\right] 、 \mathrm{Fe}^{3+}$ 、 $\mathrm{H}_{2} \mathrm{O}_{2}$ 、烟酰胺腺嘌呤二核苷酸 $(\mathrm{NADH})$ 和 $\mathrm{O}_{2}$ 等的还 原. ${ }^{10-20}$ 而 $\mathrm{K}_{3}\left[\mathrm{Fe}(\mathrm{CN})_{6}\right]$ 和 $\mathrm{O}_{2}$ 常作为 MFCs 的阴极受 体, ${ }^{1,3}$ 为此, 生物电化学工作者表现出对石墨烯及功 能化石墨烯的极大兴趣, 在过去短短三年时间里对 BESs 的石墨烯电极特别是 MFCs 的石墨烯电极开 展了广泛的研究.

\section{2 用于 MFCs 的石墨烯电极}

目前用于 MFCs 不同修饰石墨烯及基底材料的 电极有: 石墨烯纳米氧化带/碳纸、石墨烯/碳布、聚 苯胺杂化的石墨烯、石墨烯/不锈钢网、褶皱的石墨 烯/碳布、微生物还原的石墨烯、海绵-石墨烯/不锈 钢网、石墨烯/几丁质、石墨烯担载四磺酸酞菁铁 $(\mathrm{FeTsP}) /$ 碳纸、氮掺杂石墨烯/碳布、掺杂铁氮石墨 烯/碳纸、石墨烯担载二氧化锰/碳纸等(图 1), 可分为 石墨烯电极、掺杂石墨烯电极、担载石墨烯电极.

\section{1 石墨烯电极}

常规的石墨烯制备方法主要是物理法(如机械 剥离、取向附生、加热 $\mathrm{SiC}$ 、爆炸法)和化学法(如石墨 插层法、热膨胀剥离法、电化学法、化学气相沉积 法、还原石墨氧化物法、球磨法)等. ${ }^{21}$ 其中, 还原石墨 氧化物的方法是用强氧化剂将石墨氧化使石墨层 与层间引入大量含氧官能团(羟基、环氧官能团、羰 基、羧基), 增大石墨层与层间的距离, 然后超声剥离 得到石墨烯氧化物的溶液, 最后再通过化学、电化 学或生物的方法还原得到石墨烯. 由于石墨烯氧化 物可以通过氧化石墨而大量高效制备, 为石墨烯的 规模化制备提供了可能. 另外, 溶液中分散的石墨 烯氧化物可以和聚合物等其他材料形成力学和电 化学性能更优异的复合材料体系, ${ }^{22}$ 因此, 目前还原 石墨氧化物的方法在 MFCs 领域应用较多. ${ }^{11-15,17,23}$ Zhang 等 ${ }^{23}$ 用还原石墨氧化物的方法合成石墨烯, 再 将石墨烯粉用聚四氟乙烯粘在不锈钢网上构建了 较大表面积的石墨烯阳极, 为大肠杆菌的附着提供 了更多的位点; 同时, 该石墨烯具有一定的电化学 催化活性, 降低了阳极过电势, 提高电子转移效率, 获得的最大产电输出是相同条件下以不锈钢网为 阳极 MFCs 的 18 倍. 受到石墨烯氧化物还原形成石 墨烯和微生物混合物的启发, ${ }^{24,25} \mathrm{Zhou}$ 及其合作者 ${ }^{26,27}$ 利用 Shewanella 的厌氧或兼性厌氧条件下的呼吸作 用, 将石墨烯氧化物在 MFCs 阳极还原形成石墨 烯和微生物的混合物时, MFCs 的最大产电输出比 无石墨烯的阳极提高了 $32 \%{ }^{26}$ 当石墨烯氧化物在 MFCs 阴极还原形成石墨烯和微生物的混合物时, 


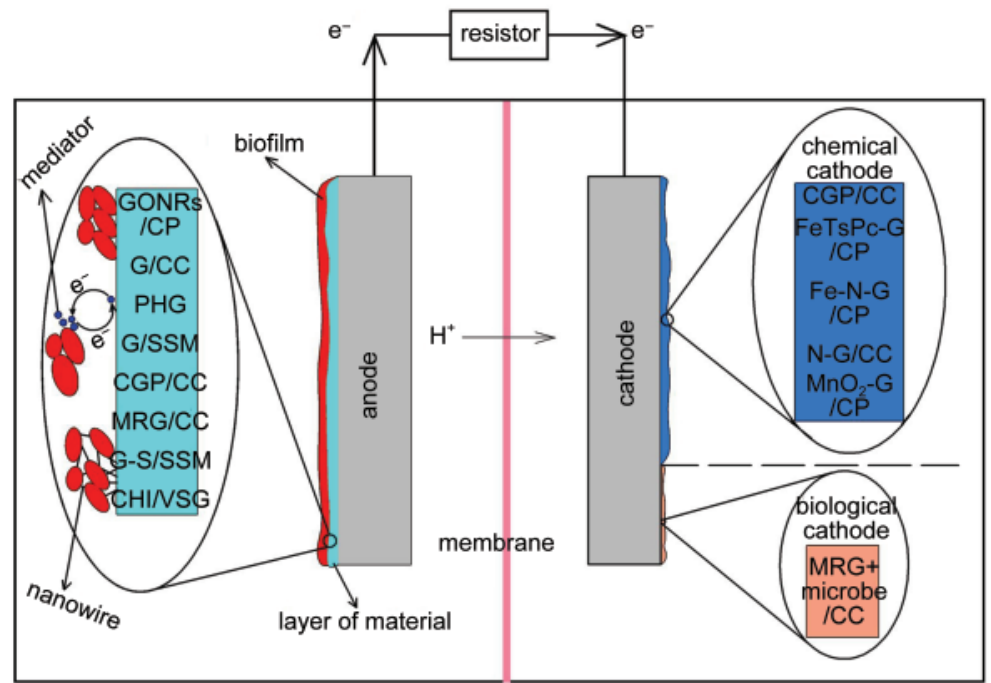

图 1 不同形貌石墨烯及其修饰电极用于微生物燃料电池示意图

Fig.1 Schematic diagram of graphene and modified graphene electrode in MFCs

G: graphene, CC: carbon cloth, SSM: stainless steel mesh, CP: carbon paper, GONRs: graphene oxide nanoribbons, PHG: polyaniline hybridized graphene, CGP: crumpled graphene particles, MRG: microbially reduced graphene,

G-S: graphene-sponges, CHI/VSG: chitosan/vacuum stripped graphene, N-G: nitrogen-doped graphene

MFCs 的最大产电比无石墨烯的阴极提高了 $103 \%$. $^{27}$ 由于高质量完美的石墨烯易通过 $\pi-\pi$ 键聚 合形成多层石墨烯, 这降低了石墨烯优良的电化学 催化性能和高电子传递效率. ${ }^{9}$ Xiao 等 ${ }^{12}$ 用气溶胶辅 助毛细压缩过程将薄膜状石墨烯变为类纸团状石 墨烯, 从而有效阻止石墨烯通过 $\pi-\pi$ 键聚合形成多
层石墨烯. 类纸团状石墨烯具有三维立体结构、表 面积更大、利于微生物附着、电子转移效率更高等 优点, 应用于 MFCs 阳极 ${ }^{13}$ 和阴极 ${ }^{12}$ 输出电能分别达 480 和 $440 \mathrm{~mW} \cdot \mathrm{m}^{-2}$. 而且, MFCs 连续运行三个月以 上, 电能输出仍保持稳定, 表明石墨烯电极具有良 好的稳定性和可重复性. 用于 MFCs 阳极 ${ }^{13,17,23,26,28-32}$

表 1 用于 MFCs 阳极的石墨烯和修饰石墨烯电极

Table 1 Graphene and modified graphene for anodic electrodes in MFCs

\begin{tabular}{|c|c|c|c|c|c|c|c|c|c|}
\hline \multirow{2}{*}{$\begin{array}{l}\text { Anode } \\
\text { materials }\end{array}$} & \multirow{2}{*}{$\begin{array}{c}\text { Anode } \\
\text { microbial }\end{array}$} & \multirow{2}{*}{$\begin{array}{c}\text { Anode } \\
\text { substrate }\end{array}$} & \multirow{2}{*}{$\begin{array}{c}\text { Reactor } \\
\text { configuration }\end{array}$} & \multirow{2}{*}{ Cathode } & \multirow{2}{*}{ Catholyte } & \multicolumn{2}{|c|}{$\begin{array}{c}\text { Maximum } \\
\text { power density }\end{array}$} & \multirow{2}{*}{$T_{\text {expt }} /{ }^{\circ} \mathrm{C}$} & \multirow{2}{*}{ Ref. } \\
\hline & & & & & & $\frac{P}{\left(\mathrm{~mW} \cdot \mathrm{m}^{-2}\right)}$ & $\frac{P}{\left(\mathrm{~W} \cdot \mathrm{m}^{-3}\right)}$ & & \\
\hline $\mathrm{CGP} / \mathrm{CC}$ & anaerobic sludge & sodium acetate & dual chamber & carbon brush & $\begin{array}{c}1 \mathrm{~mol} \cdot \mathrm{L}^{-1} \\
\mathrm{~K}_{3}\left[\mathrm{Fe}(\mathrm{CN})_{6}\right]\end{array}$ & 480 & 3.6 & 20 & 13 \\
\hline $\mathrm{G} / \mathrm{CC}$ & $\begin{array}{c}\text { Pseudomonas } \\
\text { aeruginosa }\end{array}$ & glucose & dual chamber & carbon cloth & $\begin{array}{c}50 \mathrm{mmol} \cdot \mathrm{L}^{-1} \\
\mathrm{~K}_{3}\left[\mathrm{Fe}(\mathrm{CN})_{6}\right]\end{array}$ & 52.5 & 0.07 & $\begin{array}{c}\text { not } \\
\text { provided }\end{array}$ & 17 \\
\hline $\begin{array}{l}\text { PTFE+G/ } \\
\text { SSM }\end{array}$ & $\begin{array}{c}\text { Escherichia } \\
\text { coli }\end{array}$ & glucose & dual chamber & carbon paper & $\begin{array}{c}50 \mathrm{mmol} \cdot \mathrm{L}^{-1} \\
\mathrm{~K}_{3}\left[\mathrm{Fe}(\mathrm{CN})_{6}\right]\end{array}$ & 2668 & 2.32 & 35 & 23 \\
\hline $\mathrm{MRG} / \mathrm{CC}$ & pnaerobic sludge & sodium acetate & $\begin{array}{c}\text { air-cathode } \\
\text { single-chamber }\end{array}$ & $\mathrm{Pt}+$ carbon cloth & air cathode & 1905 & 47.63 & 30 & 26 \\
\hline $\begin{array}{l}\text { GONRs/ } \\
\mathrm{CP}\end{array}$ & $\begin{array}{c}\text { Shewanella } \\
\text { oneidensis MR-1 }\end{array}$ & not provided & not provided & carbon paper & not provided & 34.2 & $\begin{array}{c}\text { not } \\
\text { provided }\end{array}$ & $\begin{array}{c}\text { not } \\
\text { provided }\end{array}$ & 28 \\
\hline G-S/SSM & previous MFC & glucose & dual chamber & $\mathrm{Pt}+$ carbon cloth & not provided & 1570 & 394 & $\begin{array}{c}\text { not } \\
\text { provided }\end{array}$ & 29 \\
\hline PHG & $\begin{array}{c}\text { Shewanella } \\
\text { oneidensis MR-1 }\end{array}$ & lactate & dual chamber & carbon cloth & $\begin{array}{c}50 \mathrm{mmol} \cdot \mathrm{L}^{-1} \\
\mathrm{~K}_{3}\left[\mathrm{Fe}(\mathrm{CN})_{6}\right]\end{array}$ & 768 & $\begin{array}{c}\text { not } \\
\text { provided }\end{array}$ & $\begin{array}{c}\text { not } \\
\text { provided }\end{array}$ & 30 \\
\hline $\mathrm{PHG} / \mathrm{CC}$ & anaerobic sludge & sodium acetate & dual chamber & carbon flets & $\begin{array}{c}50 \mathrm{mmol} \cdot \mathrm{L}^{-1} \\
\mathrm{~K}_{3}\left[\mathrm{Fe}(\mathrm{CN})_{6}\right]\end{array}$ & 1390 & 22.52 & 30 & 31 \\
\hline $\mathrm{CHI} / \mathrm{VSG}$ & $\begin{array}{c}\text { Pseudomonas } \\
\text { aeruginosa }\end{array}$ & glucose & dual chamber & not provided & not provided & 1530 & $\begin{array}{c}\text { not } \\
\text { provided }\end{array}$ & $\begin{array}{c}\text { not } \\
\text { provided }\end{array}$ & 32 \\
\hline
\end{tabular}


表 2 用于 MFCs 阴极的石墨烯和修饰石墨烯电极

Table 2 Graphene and modified graphene for cathodic electrodes in MFCs

\begin{tabular}{|c|c|c|c|c|c|c|c|}
\hline \multirow{2}{*}{ Cathode } & \multirow{2}{*}{ Electron acceptor } & \multirow{2}{*}{ Reactor configuration } & \multirow{2}{*}{ Anode material } & \multicolumn{2}{|c|}{ Power density } & \multirow{2}{*}{$T_{\text {expt }} /{ }^{\circ} \mathrm{C}$} & \multirow{2}{*}{ Ref. } \\
\hline & & & & $P /\left(\mathrm{mW} \cdot \mathrm{m}^{-2}\right)$ & $P /\left(\mathrm{W} \cdot \mathrm{m}^{-3}\right)$ & & \\
\hline $\mathrm{CGP} / \mathrm{CC}$ & $\mathrm{O}_{2}$ & dual chamber & carbon cloth & 440 & 3.3 & 20 & 12 \\
\hline $\mathrm{Fe}-\mathrm{N}-\mathrm{G} / \mathrm{CP}$ & $\mathrm{O}_{2}$ & single chamber & carbon felt & 1150 & 71.9 & 30 & 13 \\
\hline FeTsPc-G/CP & $\mathrm{O}_{2}$ & dual chamber & carbon paper & 817 & 5.7 & 35 & 14 \\
\hline $\mathrm{MnO}_{2}-\mathrm{G} / \mathrm{CP}$ & $\mathrm{O}_{2}$ & single chamber & carbon felt & 2084 & 10.4 & 24 & 15 \\
\hline $\mathrm{G} / \mathrm{CC}$ & $\mathrm{O}_{2}$ & dual chamber & Carbon cloth & 323 & 16.2 & 30 & 27 \\
\hline $\mathrm{N}-\mathrm{G} / \mathrm{CC}$ & $\mathrm{O}_{2}$ & single chamber & carbon fiber brush & 1350 & not provided & 25 & 33 \\
\hline
\end{tabular}

和阴极 ${ }^{12-15,27,33}$ 的石墨烯和修饰石墨烯电极材料分别 列于表 1 和表 2 .

选择良好的石墨烯基底材料, 也是提高 MFCs 性能的有效途径. Huang 等 ${ }^{28}$ 用化学方法将多壁碳 纳米管剪开, 形成氧化石墨烯纳米带, 然后用电泳 沉积法将其负载到碳纸上, 此石墨烯/碳纸阳极的 电化学活性表面积是无石墨烯修饰的 1.5 倍, 纯菌 Shewanella oneidensis MR-1 或混菌催化下的最大电 能输出均是后者的 4 倍. 石墨烯理论表面积约为 $2600 \mathrm{~m}^{2} \cdot \mathrm{g}^{-1}$, 由于石墨烯薄膜的聚集作用使实验中 得到的石墨烯并非单层结构, 表面积会降低. Zhang 等 ${ }^{23}$ 用还原石墨氧化物方法得到的石墨烯表面积仅 为 $264 \mathrm{~m}^{2} \cdot \mathrm{g}^{-1}$. 为了弥补石墨烯聚集导致的表面积 减小, Liu 等 ${ }^{17}$ 以碳布为基底, 借助碳布的多孔结构, 采用电化学沉积将石墨烯形成三维多孔的立体结 构, 提高了系统输出电能. 与石墨烯/碳布电极相似, 将海绵在石墨烯溶液中重复浸泡、晾干后得到石墨 烯海绵, 为了补偿海绵的低电导率, 将石墨烯海绵 粘贴在不锈钢网上, 从而提高电能输出. ${ }^{29}$ 然而, 该 MFCs 单位体积的电能输出仅为 $0.07 \mathrm{~W} \cdot \mathrm{m}^{-3}$, 远低于 目前其它材料的 MFCs 电能输出(表 1). Gurunathan 等 ${ }^{34}$ 发现石墨烯及石墨烯氧化物对铜绿假单胞菌有 氧化应激介导的抗菌活性; 此外, 石墨烯及石墨烯 氧化物的大小对细胞的毒性也有影响, 且高浓度石 墨烯及石墨烯悬浮液对细胞的毒性更大. 因此, 这 种石墨烯电极的较低电能输出可能归因于电极材 料对电活性微生物的毒性. 考虑到石墨烯基底材料 对 PC12 细胞和 Osteoblasts 细胞的无毒副作用, ${ }^{35}$ 深 入开展石墨烯类电极材料对 MFCs 电活性微生物的 毒性评价也是拓展石墨烯在 MFCs 领域应用的重要 方面.

目前, 关于石墨烯提高 MFCs 电能输出的机制 还存在很多争议. 石墨烯/碳布阳极附着的铜绿假单 胞菌可分泌绿皮菌素, 并以此作为电子介体传递电
子. ${ }^{17}$ 与不含石墨烯的碳布对照相比, 石墨烯存在下 的阳极液中绿皮菌素浓度是前者的 3 倍, 石墨烯较 高的生物相容性可能促进了电极表面电活性微生 物的生长, 不仅为直接电子传递提供了更多的活性 位点, 而且也刺激了铜绿假单胞菌分泌更多的绿皮 菌素介体. ${ }^{17}$ 但笔者认为, 绿皮菌素介体量应该与生 物量正相关, 石墨烯/碳布阳极可能通过促进微生物 生长和繁殖, 间接提高了绿皮菌素介体在阳极液中 含量. 实际上, 刺激更多介体分泌的作用并不仅限 于石墨烯, 石墨阳极也能促进纯菌 Shewanella loihi$c a$ PV-4 分泌核黄素, 从而使 MFCs 的电子传递随着 运行时间的延长由直接电子传递逐渐变为间接电 子传递. ${ }^{36}$ 除绿皮菌素介体外, 石墨烯/碳布阳极中还 发现有未知结构的代谢产物, 此产物的介体传递作 用及其与石墨烯的关系还有待深入探究. ${ }^{17}$ 也有研 究认为, 通过碳纳米管制得的石墨烯纳米氧化带具 有较大的长径比, 起到电活性菌的类纳米线作用; 且电极表面电荷转移电阻显著减小, 反应活化能降 低, 电子转移速率提高; 28,37 而石墨烯较大的表面积 和边缘结构以及大量的功能基团也是电能输出提 高的重要原因..$^{38}$

\section{2 掺杂石墨烯电极}

电化学催化过程一般需经历底物吸附、电极表 面氧化还原和产物扩散三个步骤. 石墨烯的共轭结 构能够加强电化学催化过程对底物的吸附能力, 从 而促进电化学催化. ${ }^{9}$ 掺杂了 $N 、 P 、 S 、 B 、 S e 、 I$ 的石墨 烯对氧的还原反应(ORR)具有催化作用, 使氧的 ORR 的起始电势升高. 目前, 实现石墨烯氮掺杂的 方法主要有化学气相沉积 $(C V D)$ 法、 $\mathrm{N}_{2}$ 等离子处理 法、电弧放电法、高能电热法、模板法等, 其中 CVD 法应用最为广泛. ${ }^{39}$ Feng 等 ${ }^{33}$ 用 CVD 法以氧脲酰氯 和苦味酸在 $320^{\circ} \mathrm{C} 、 30 \mathrm{MPa}$ 条件反应合成了适于 BESs 的氮掺杂率为 $12.5 \%$ 的克级氮载石墨烯. 该 $\mathrm{O}_{2}$ 受体氮载石墨烯阴极 MFCs 输出电能为 $1350 \mathrm{~mW}$. 
$\mathrm{m}^{-2}$ (表 2$)$, 与 $\mathrm{Pt} / \mathrm{C}$ 阴极 $\left(1420 \mathrm{~mW} \cdot \mathrm{m}^{-2}\right)$ 相近, 表明氮 载石墨烯较强的催化作用. 但合成氮载石墨烯的出 发底物是氰腿酰氯和苦味酸等剧毒和易爆炸化学 品; 且氮载石墨烯的催化是两电子和四电子的竞争 过程, 系统库仑效率较低; 同时两电子过程形成的 $\mathrm{H}_{2} \mathrm{O}_{2}$ 产物还会腐蚀反应器和电极. Lai 等 ${ }^{40}$ 认为, 氮 载石墨烯中氮主要以石墨氮、吡啶型氮和吡咯型氮 形式存在, 其中, 石墨氮和吡啶型氮含量决定氮载 石墨烯的氧还原反应催化效果, 石墨氮含量影响极 限电流密度, 较多的吡啶型氮能提高氧还原反应的 起始电势, 但总氮含量对氧还原反应的催化无明显 影响. 同样, 用铁氮功能化的石墨烯(Fe-N-G)为阴极 催化氧的氧还原反应, 铁在石墨烯的层与层间能够 阻止石墨烯的聚集, 使掺杂了氮的石墨烯更稳定, 并快速催化两电子过程形成的 $\mathrm{H}_{2} \mathrm{O}_{2}$ 歧化为水, 使 $\mathrm{Fe}-\mathrm{N}-\mathrm{G}$ 的催化以四电子模式进行. ${ }^{13}$ 从电负性方面 看, Fe-N-G 中杂化的氮原子(石墨型氮、吡啶型氮、 吹咯型氮)带有负电荷, 能够改善石墨烯吸收氧气, 并减弱 $\mathrm{O}-\mathrm{O}$ 键能, 利于氧的氧还原反应进行, 从而 提高电能输出. ${ }^{13}$ 而 $\mathrm{Fe}-\mathrm{N}-\mathrm{G}$ 中不同类型氮含量将不 同程度地改善石墨烯吸收氧气, 从而 $\mathrm{O}-\mathrm{O}$ 键能弱 化程度不同, 电能输出不同. 因此, 还应该深入考察 不同比例 Fe、N、C 条件下的 Fe-N-G 催化效果, 这将 有助于剖析 MFCs 的阴极催化机理, 优化系统性能. 与铁氮石墨烯相同, $\mathrm{Co} 、 \mathrm{Ni}$ 等金属氮载石墨烯也是 一种高电化学活性和经济有效的催化剂, 它们能催 化氧的氧还原反应, 提高系统性能.

合成氮载石墨烯的原料决定产物中氮的存在 类型和含量, 进而影响氧的 ORR 催化性能. 氨气、吡 啶、乙腈、三聚氰胺、尿素等含氮化合物及氮等离子 体常作为氮源使用. 其中, 尿素是一种高氮含量试 剂, 易溶于水、毒性小、容易处理、利于环保, 并且显 示出良好的还原能力, 可用来制备氮掺杂石墨烯. ${ }^{39}$ Liu等 ${ }^{41}$ 以氧化石墨烯为原料、尿素为还原剂和氮掺 杂剂, 合成了以吡啶型氮为主, 氮含量约为 $4 \%$ 的氮 载石墨烯, 其在碱性或酸性条件下对氧的 ORR 的催 化效果与商业化的 $\mathrm{Pt} / \mathrm{C}$ 相同或相近. 与氮载石墨烯 相似, Yang 等 ${ }^{19}$ 指出硫掺杂石墨烯在碱性条件下对 氧的 ORR 的催化效果好于商业化 $\mathrm{Pt} / \mathrm{C}$, 为其在空气 阴极 MFCs 的应用提供了可能.

\section{3 担载石墨烯电极}

\subsection{1 石墨烯担载金属及金属氧化物}

以空气为阴极电子受体的 MFCs 由于来源简便
易得、产物清洁而被广泛研究, 但氧在中性环境下 的电极反应过电势较高, 使电能输出较低. 铂基催 化剂是有效降低反应过电势、提高系统性能的理想 催化剂. 然而, 高成本和可能的二次污染是制约其 规模化应用的瓶颈. ${ }^{2}$ 非铂催化剂如过渡金属镍 ${ }^{42}$ 及 其氧化物、卟啉和酞菁、聚吡咯、氧化铅、石墨颗粒 以及活性炭纤维毡, 均能不同程度地降低氧受体的 ORR 过电势, 但催化效率均不及铂基催化剂. ${ }^{3}$ 近期 的研究结果表明, 石墨烯薄膜也能催化氧的 ORR, 而石墨烯高的导电性还能降低电池内阻, 增加电能 输出, 从而为空气阴极 MFCs 的规模化放大提供了 可能. ${ }^{11}$ 因此, 若能在石墨烯薄膜表面担载铂, 将可 能在保持催化性能的同时, 减少铂使用量. 王万丽 和马紫峰 ${ }^{43}$ 用嗍氢化钠共还原法制备 40\%(w/w) 铂/ 石墨烯电催化剂. 该催化剂对氧的 ORR 活性较铂/ 碳催化剂差, 但稳定性有所提高. 与此不同, 石墨烯 担载铂催化甲醇的氧化反应优于炭黑载铂催化 剂. ${ }^{22}$ 另外, 金属如 $\mathrm{Co} 、 \mathrm{Cu} 、 \mathrm{Mn} 、 \mathrm{Fe} 、 \mathrm{Ni}$ 及其氧化物对 氧的 ORR 催化效果不及铂基催化剂, 但前者来源丰 富, 价格低廉, 与石墨烯形成的复合物对氧的 ORR 表现出比单一催化剂更优异的催化性能. ${ }^{9}$ 基于 $\beta-\mathrm{MnO}_{2}$ 晶型在空气阴极 MFCs 中对氧的良好催化 作用, ${ }^{44}$ 将高锰酸钾与石墨烯纳米薄膜(GNS) 在微波 加热条件下, 形成以非共价键均匀复合的 $\beta-\mathrm{MnO}_{2} /$ GNS(比表面积 $158 \mathrm{~m}^{2} \cdot \mathrm{g}^{-1}$ ), 与 $\mathrm{MnO}_{2}$ 阴极相比, 该 $\beta-\mathrm{MnO}_{2} / \mathrm{GNS}$ 的 ORR 催化效果更好, 且电极的峰电 流正比于扫描速率的平方根, 说明 $\beta-\mathrm{MnO}_{2} / \mathrm{GNS}$ 催 化的 ORR 是扩散控制过程. 与 $\mathrm{MnO}_{2}$ 阴极(比表面积 $\left.41 \mathrm{~m}^{2} \cdot \mathrm{g}^{-1}\right) \mathrm{MFCs}$ 相比, 前者电能输出提高 $614 \mathrm{~mW}$. $\mathrm{m}^{-2}$ (表 2). ${ }^{15} \mathrm{Liang}$ 等 ${ }^{45}$ 证明, 石墨烯担载 $\mathrm{Co}_{3} \mathrm{O}_{4}$ 在碱 性条件下对氧的 ORR 催化与商品化 $\mathrm{Pt} / \mathrm{C}$ 相同, 均为 四电子过程. 与石墨烯担载 $\mathrm{Co}_{3} \mathrm{O}_{4}$ 相比, 氮掺杂石墨 烯担载 $\mathrm{Co}_{3} \mathrm{O}_{4}$ 提高氧的 $\mathrm{ORR}$ 起始还原电位, 增加峰 电流, 表明后者更好的导电性能. 此外, 谢鹏洋等 ${ }^{30}$ 通过密度泛函理论研究了 $\mathrm{Ag} 、 \mathrm{Au} 、 \mathrm{Pt}$ 原子在完美和 点缺陷(包括 $\mathrm{N}$ 掺杂、 $\mathrm{B}$ 掺杂、空位点缺陷)石墨烯上 的吸附及其界面性质, 表明 $\mathrm{Ag} 、 \mathrm{Au}$ 不能在完美的石 墨烯上吸附; N、B 掺杂增强了三种金属与石墨烯之 间的相互作用; 而空位点缺陷诱发三种金属在石墨 烯上具有强化学吸附作用. 通过对材料表面电子结 构分析发现, N 掺杂增强了 $\mathrm{Au} 、 \mathrm{Pt}$ 与 $\mathrm{C}$ 形成的共价 键, 而 $\mathrm{Au} 、 \mathrm{Ag}$ 与 $\mathrm{B}$ 形成了化学键. 空位点缺陷不仅 是金属原子的几何固定点, 同时也增加了金属原子 
和碳原子之间的成键. 综上, 我们有理由相信, 掺杂 石墨烯和担载石墨烯的复合物电极在 MFCs 中会表 现出比单一石墨烯电极更优异的电化学催化性能.

\subsection{2 其它担载石墨烯电极}

石墨烯规整的二维平面结构使其可作为理想 的模板担载催化剂. 就 MFCs 而言, 阳极靠产电细菌 消耗有机物产生电能, 附着于阳极的电活性微生 物越多越利于产电, 因此, 在石墨烯上担载细胞相 容的物质会有利于产电细菌的附着. 目前已用于 MFCs 阳极的石墨烯担载物有聚苯胺和几丁质. Yong 等 ${ }^{46}$ 通过化学气相沉积法将石墨烯负载到泡沫 镍上, 进而将聚苯胺原位聚合在石墨烯上, 再用盐 酸将镍骨架腐蚀掉, 形成聚苯胺杂化的三维石墨烯 泡沫阳极, 此电极比一般碳材料表面积更大、附着 的微生物更多. 由于该石墨烯泡沫的阳极液中电子 介体核黄素量无明显增加, 较高的电能输出主要归 因于较大的材料表面积提供了更多的电子传递活 性位点. 阻抗分析进一步验证了此种材料的较高电 荷转移效率. Hou 等 ${ }^{31}$ 用电化学还原石墨烯氧化物 的方法得到石墨烯, 然后再在聚苯胺溶液中浸泡 12 $\mathrm{h}$ 获得石墨烯担载聚苯胺/碳布阳极, 得到的最大功 率密度是相同条件下碳布阳极的 3 倍(表 1 ).

从电子传递过程看, 电子由电活性细胞的呼吸 链传递到阳极电极的过程, 是通过不同的氧化还原 位点非连续式地“跳跃”传递. 故在石墨烯上杂化氧 化还原活性位点, 使其与阳极微生物形成复合物, 从而起到类似微生物间 “纳米线” 的作用, 以提高电 子传递效率, 增加电能输出. ${ }^{77}$ 然而, 石墨阳极也能 促进纯菌 Shewanella loihica PV-4 分泌核黄素, 使 MFCs 电子传递随着运行时间的延长由直接电子传 递逐渐变为间接电子传递. ${ }^{36}$ 据此, 若将相应的间接 电子传递的介质固定在石墨烯上, 不仅能促进电子 传递, 而且能避免因阳极液的流失使介体流失.

不同合成方法得到的石墨烯担载复合物的表 面积差异很大, 影响 MFCs 的电能输出. 用冰隔离诱 导自组装的方法将几丁质/真空剥离的石墨烯溶液 在液氮中冷却后干燥, 形成三维结构的多孔的、树 枝状的几丁质/真空剥离石墨烯泡沫, 比表面积为 $248 \mathrm{~m}^{2} \cdot \mathrm{g}^{-1}$, 在铜绿假单胞菌催化下的电能输出达 $1530 \mathrm{~mW} \cdot \mathrm{m}^{-2}$ (表 1$)$. $^{32}$ 而 Cheng 等 ${ }^{48}$ 将壳聚糖溶液加 到氧化石墨烯溶液中, 搅拌 $10 \mathrm{~h}$ 后过滤, 再在真空 状态下干燥, 之后在氩气流下加热到 $150^{\circ} \mathrm{C}$, 保持 $45 \mathrm{~min}$, 得到石墨烯担载几丁质的三维复合物, 比表
面积达 $603 \mathrm{~m}^{2} \cdot \mathrm{g}^{-1}$. 同样, 四磺酸酞菁铁与石墨烯通 过非共价键杂化制备的FeTsPc-G 阴极, 电能输出达 $817 \mathrm{~mW} \cdot \mathrm{m}^{-2}$, 接近于 $\mathrm{Pt} / \mathrm{C}$ 阴极的电能输出 $(856$ $\mathrm{mW} \cdot \mathrm{m}^{-2}$ ); 而对照组的 FeTsPc 阴极电能输出仅为 $523 \mathrm{~mW} \cdot \mathrm{m}^{-2}$. ${ }^{14}$ 石墨烯担载氧的 ORR 催化剂, 不仅 得到了与 $\mathrm{Pt} / \mathrm{C}$ 催化剂相当的催化效果, 而且, 石墨 烯和其它非贵金属催化剂的价格低廉, 来源丰富, 为 MFCs 的大规模应用提供了可能. 另外, Ahmed 和 Jeon $^{16}$ 证明了石墨烯担载三十二烷基甲基氯化铵对 氧的 ORR 催化作用. 将石墨烯作为 MFCs 阴极氧的 ORR 催化剂的基底, 不仅能增强其催化效果, 而且 能降低 MFCs 成本. 与担载石墨烯电极不同, 以电活 性微生物为催化剂的氧受体生物阴极以其清洁、可 持续性等优点引起人们的关注. ${ }^{49}$ 结合石墨烯电极 的氧受体生物阴极 MFCs 研究目前还少有报道, ${ }^{25}$ 石 墨烯材料表面结构及性质与电活性微生物间作用 的特殊性和普遍性规律都还不甚清楚, 相关科学问 题的解决将拓展石墨烯应用的新领域.

\section{3 展 望}

与石墨烯在 MFCs 中的广泛研究不同, 石墨烯 在 MECs 中的应用还鲜有报道. 通常认为, MECs 是 转化废物和废水生成清洁氢能的装置. ${ }^{50-52}$ 在影响 MECs 产氢的诸多因素(微生物种类、底物类型、膜 材料、溶液化学、电极材料)中, 电极材料是影响产氢 效率、决定反应器成本的重要方面. ${ }^{53-55}$ 理想的 MECs 电极应具有较大的比表面积、最小的极化损 失、良好的导电性能, 从而使电路中电流密度最大、 产氢效率最高. 碳纳米管电极能催化产氢, ${ }^{53}$ 不锈钢 网、 ${ }^{54}$ 碳布担载镍粉、碳布担载锄镍氧化物 $\left(\mathrm{Ni}_{6} \mathrm{MoO}_{3}\right) 、{ }^{56}$ 不锈钢网担载二硫化钿 ${ }^{57}$ 的产氢量与 碳布担载铂的效果相当, 为规模化、低成本的 MECs 产氢提供了可能. 对革兰氏阴性菌Sporomusa ovata 利用 $\mathrm{CO}_{2}$ 合成乙酸的生物阴极 MECs 而言, 分别用 壳聚糖、氧尿酰氯、3-氨基丙基三乙氧基硅烷、聚苯 胺等修饰碳布阴极, 使其表面带正电荷, 能有效改 善菌细胞与电极间作用, 提高乙酸产量 3-7 倍; 当用 纳米级金属颗粒如金、钯、镍修饰电极表面能提高 乙酸产量 4.5-6.0 倍; 以棉布或聚酯纤维为基底、碳 纳米管修饰的电极比碳布电极的乙酸产量提高 3 倍. ${ }^{58}$ 因此, 调整和改变阴极表面电势, 使其与菌细 胞电子传递介体间的作用最佳, 是定向优化和提高 生物阴极 MECs 性能的有效途径. 石墨烯具有降低 
MFCs 过电势的作用, 且催化效果好于碳纳米管, 而 石墨烯在 MECs 中的应用, 特别是定向产品制备下 的石墨烯作用还远未引起重视. 有理由相信, MECs 合成化学品研究的不断丰富和发展, 将为石墨烯的 应用提供新的途径和机遇.

BESs 技术的清洁、高效、可持续性已引起了环 境、材料、微生物、清洁能源等各领域科学家的广泛 兴趣. 石墨烯优异的导电性、良好的电催化性能使 其在很多方面得到了应用, 作为电极材料应用于 MECs 的研究目前还是空白, 应用于 MFCs 的研究也 仅限于提高电能的探索, 推测的电子传递机制仅为 “石墨烯较大的表面积利于更多的微生物附着, 优 异的导电性利于电子传递”. 实际上, 石墨烯与传统 石墨的结构和性能不同, 电活性微生物作用下的石 墨烯电极的电子传递是否具有某些特殊性, 还有待 于进一步探索. 通常认为, 制约电子传递的关键是 电极与电活性微生物间的作用. ${ }^{2}$ 利用基因消除技 术, 研究和表征石墨烯或石墨烯不同修饰电极与模 板电活性菌 Geobacter 或 Shewanella 间的作用, 是探 讨石墨烯的 BESs 电子传递机理、提高系统性能的 重要方面. 其次, 寻找有效方法跟踪石墨烯的 BESs 电子传递, 可能是直观、形象地表征电子传递的理 想选择; 再次, 结合 BESs 的不同功用, 特别是合成 化学品的 BESs 的不同要求, 定向设计和制备特殊 功用的石墨烯, 必将拓展石墨烯应用的领域和范 围. 最后, 与目前 BESs 研究缺乏统一的国际标准类 似, ${ }^{59}$ 石墨烯的 BESs 研究也面临同样的问题. 由于 BESs 的运行性能受多种因素影响, 如电极材料、阳 极和阴极反应、温度、溶液电导率和缓冲体系类型、 反应器构型、接种和驯化方式等, 不同实验室不同 条件下的石墨烯 BESs 运行结果缺少可比性, 研究 结果的标准化是当前该领域面临的主要问题之一. 可以预计, 随着 BESs 研究的深入和应用领域的拓 展以及新型石墨烯制备技术的发展，石墨烯应用于 BESs 及其相关学科的研究将越来越受到人们的重 视.

\section{References}

(1) Logan, B. E. Nat. Rev. Microbiol. 2009, 7 (5), 375. doi: 10.1038/ nrmicro2113

(2) Logan, B. E. Appl. Microbiol. Biotechnol. 2010, 85 (6), 1665. doi: 10.1007/s00253-009-2378-9

(3) Logan, B. E.; Rabaey, K. Science 2012, 337 (6095), 686. doi: 10.1126/science. 1217412
(4) Wei, J.; Liang, P.; Huang, X. Bioresour. Technol. 2011, 102 (20), 9335. doi: 10.1016/j.biortech.2011.07.019

(5) Zhou, M. H.; Chi, M. C.; Luo, J. M.; He, H. H.; Jin, T. J. Power Sources 2011, 196 (10), 4427. doi: 10.1016/j.jpowsour. 2011.01 .012

(6) Jiang, L. L.; Lu, X. J. Funct. Mater. 2012, 23 (43), 2881. [姜丽 丽, 鲁 雄. 功能材料, 2012, 23 (43), 2881.]

(7) Sun, Y. Q.; Wu, Q.; Shi, G. Q. Energy Environ. Sci. 2011, 4 (4), 1113. doi: $10.1039 / \mathrm{c} 0 \mathrm{ee} 00683 \mathrm{a}$

(8) Wang, H.; Hu, Y. H. Energy Environ. Sci. 2012, 5 (8), 8182. doi: $10.1039 / \mathrm{c} 2 \mathrm{ee} 21905 \mathrm{k}$

(9) Huang, C. C.; Li, C. H.; Shi, G. Q. Energy Environ. Sci. 2012, 5 (10), 8848. doi: 10.1039/c2ee22238h

(10) Hu, Y. J.; Jin, J.; Zhang, H.; Wu, P.; Cai, C. X. Acta Phys. -Chim. Sin. 2010, 26 (8), 2073. [胡耀娟, 金 娟, 张 卉, 吴 萍, 蔡称心. 物理化学学报, 2012, 26 (8), 2073.] doi: 10.3866/PKU.WHXB20100812

(11) Wu, J. J.; Wang, Y.; Zhang, D.; Hou, B. R. J. Power Sources 2011, 196 (3), 1141. doi: 10.1016/j.jpowsour.2010.07.087

(12) Xiao, L.; Damien, J.; Luo, J. Y.; Jang, H. D.; Huang, J. X.; He, Z. J. Power Sources 2012, 208 (1), 187.

(13) Li, S. Z.; Hu, Y. Y.; Xu, Q.; Sun, J.; Hou, B.; Zhang, Y. P. J. Power Sources 2012, 213 (1), 265.

(14) Zhang, Y. Z.; Mo, G. Q.; Li, X. W.; Ye, J. S. J. Power Sources 2012, 197 (1), 93.

(15) Wen, Q.; Wang, S. Y.; Yan, J.; Cong, L. J.; Pan, Z. C.; Ren, Y. M.; Fan, Z. G. J. Power Sources 2012, 216 (1), 187.

(16) Ahmed, M. S.; Jeon, S. J. Power Sources 2012, 218 (1), 168.

(17) Liu, J.; Qiao, Y.; Guo, C. X.; Lim, S.; Song, H.; Li, C. M. Bioresour. Technol. 2012, 114 (1), 275.

(18) Palaniselvam, T.; Aiyappa, H. B.; Kurungot, S. J. Mater. Chem. 2012, 22 (45), 23799. doi: 10.1039/c2jm35128e

(19) Yang, Z.; Yao, Z.; Fang, G. Y.; Nie, H. G.; Liu, Z.; Zhou, X. M.; Chen, X. A.; Huang, S. M. J. Am. Chem. Soc. 2012, 6 (1), 205.

(20) Wu, J. J.; Zhang, D.; Wang, Y.; Wan, Y.; Hou, B. R. J. Power Sources 2012, 198 (1), 122.

(21) Shi, Y. S.; Li, X. H.; Ning, Q. J. Electron. Compon. Mater. 2010, 29 (8), 70 . [史永胜, 李雪红, 宁青菊. 电子元件与材料, 2010, $29(8), 70$.

(22) Fu, Q.; Bao, X. H. Chin. Sci. Bull. 2009, 54 (18), 2657. [傅 强,包信和. 科学通报, 2009, 54 (18), 2657.] doi: 10.1360/ 972009-1537

(23) Zhang, Y. Z.; Mo, G. Q.; Li, X. W.; Zhang, W. D.; Zhang, J. Q.; Ye, J. S.; Huang, X. D.; Yu, C. Z. J. Power Sources 2011, 196 (13), 5402. doi: 10.1016/j.jpowsour.2011.02.067

(24) Salas, E. C.; Sun, Z.; Luttge, A.; Tour, J. M. ACS Nano 2010, 4 (8), 4852. doi: 10.1021/nn101081t

(25) Wang, G. M.; Qian, F.; Saltikov, C. W.; Jiao, Y. Q.; Li, Y. Nano Res. 2011, 4, 563. doi: 10.1007/s12274-011-0112-2

(26) Yuan, Y.; Zhou, S. G.; Zhao, B.; Zhuang, L.; Wang, Y. Q. Bioresour. Technol. 2012, 116 (1), 453. 
(27) Zhuang, L.; Yuan, Y.; Yang, G. Q.; Zhou, S. G. Electrochem. Commun. 2012, 21 (1), 69.

(28) Huang, Y. X.; Liu, X. W.; Xie, J. F.; Sheng, G. P.; Wang, G. Y.; Zhang, Y. Y.; Xu, A. W.; Yu, H, Q. Chem. Commun. 2011, 47 (20), 5795. doi: 10.1039/c1cc10159e

(29) Xie, X.; Yu, G. H.; Liu, Z. N.; Criddle, C. S.; Cui, Y. Energy Environ. Sci. 2012, 5 (5), 6862. doi: 10.1039/c2ee03583a

(30) Xie, P. Y.; Zhuang, G. L.; Lü, Y. A.; Wang, J. G.; Li, X. N. Acta Phys. -Chim. Sin. 2012, 28 (2), 331. [谢鹏洋, 庄桂林, 吕永 安, 王建国, 李小年. 物理化学学报, 2012, 28 (2), 331.] doi: 10.3866/PKU.WHXB201111021

(31) Hou, J. X.; Liu, Z. L.; Zhang, P. Y. J. Power Sources 2013, 224 (1), 139.

(32) He, Z. M.; Liu, J.; Qiao, Y.; Li, C. M.; Tan, T. T. Y. Nano Lett. 2012, 12 (9), 4738. doi: 10.1021/n1302175j

(33) Feng, L. Y.; Chen, Y. G.; Chen, L. ACS Nano 2011, 5 (12), 9611. doi: $10.1021 / \mathrm{nn} 202906 \mathrm{f}$

(34) Gurunathan, S.; Han, J. W.; Dayem, A. A.; Eppakayala, V.; Kim, J. N. Int. J. Nanomed. 2012, 7 (1), 5901.

(35) Agarwal, S.; Zhou, X. Z.; Ye, F.; He, Q. Y.; Chen, G. C. K.; Soo, J.; Boey, F.; Zhang, H.; Chen, P. Langmuir 2010, 26 (4), 2244. doi: $10.1021 / 1$ a9048743

(36) Jain, A.; Zhang, X. M.; Pastorella, G.; Connolly, J. O.; Barry, N.; Woolley, R.; Krishnamurthy, S.; Marsili, E. Bioelectrochemistry 2012, 87 (Suppl. 1), 28.

(37) Qiao, Y.; Bao, S. J.; Li, C. M.; Cui, X. Q.; Lu, Z. S.; Guo, J. ACS Nano 2008, 2 (1), 113. doi: 10.1021/nn700102s

(38) Zuo, X.; He, S.; Li, D.; Peng, C.; Huang, Q.; Song, S.; Fan, C. Langmuir 2010, 26 (3), 1936. doi: 10.1021/1a902496u

(39) Su, P.; Guo, H. L.; Peng, S.; Ning, S. K. Acta Phys. -Chim. Sin. 2012, 28 (11), 2745. [苏 鹏, 郭慧林, 彭 三, 宁生科. 物理 化学学报, 2012, 28 (11), 2745.] doi: 10.3866/PKU. WHXB201208221

(40) Lai, L. F.; Potts, J. R.; Zhan, D.; Wang, L.; Poh, C. K.; Tang, C. H.; Gong, H.; Shen, Z. X.; Lin, J. Y.; Ruoff, R. S. Energy Environ. Sci. 2012, 5 (7), 7936. doi: 10.1039/c2ee21802j

(41) Liu, Q.; Zhang, H. Y.; Zhong, H. W.; Zhang, S. M.; Chen, S. L. Electrochim. Acta 2012, 81 (1), 313.

(42) Wen, Q.; Liu, Z. M.; Chen, Y.; Li, K. F.; Zhu, N. Z. Acta Phys. -Chim. Sin. 2008, 24 (6), 1063. [温 青, 刘智敏, 陈 野, 李凯峰, 朱宁正. 物理化学学报, 2008, 24 (6), 1063.] doi: 10.3866/PKU.WHXB20080626

(43) Wang, W. L.; Ma, Z. F. Acta Phys. -Chim. Sin. 2012, 28 (12),
2879. [王万丽, 马紫峰. 物理化学学报, 2012, 28 (12), 2879.] doi: 10.3866/PKU.WHXB201209252

(44) Zhang, L. X.; Liu, C. S.; Zhuang, L.; Li, W. S.; Zhou, S. G. Biosens. Bioelectron. 2009, 24 (9), 2825. doi: 10.1016/j.bios. 2009.02.010

(45) Liang, Y. Y.; Li, Y. G.; Wang, H. L.; Zhou, J. G.; Wang, J.; Regier, T.; Dai, H. J. Nat. Mater. 2011, 10 (10), 780. doi: $10.1038 /$ nmat 3087

(46) Yong, Y. C.; Dong, X. C.; Mary, B. C. P.; Song, H.; Chen, P. ACS Nano 2012, 6 (3), 2394. doi: 10.1021/nn204656d

(47) Pirbadian, S.; EI-Naggar, M. Y. Phys. Chem. Chem. Phys. 2012, 14 (40), 13802. doi: 10.1039/c2cp41185g

(48) Cheng, J. S.; Du, J.; Zhu, W. J. Carbohyd. Polym. 2012, 88 (1), 61. doi: 10.1016/j.carbpol.2011.11.065

(49) Huang, L. P.; Regan, J. M.; Quan, X. Bioresour. Technol. 2011, 102 (1), 316. doi: 10.1016/j.biortech.2010.06.096

(50) Liu, H.; Grot, S.; Logan, B. E. Environ. Sci. Technol. 2005, 39 (11), 4317. doi: 10.1021/es050244p

(51) Rozendal, R. A.; Hamelers, H. V. M.; Euverink, G. J. W.; Metz, S. J.; Buisman, C. J. N. Int. J. Hydrog. Energy 2006, 31 (12), 1632. doi: 10.1016/j.ijhydene.2005.12.006

(52) Logan, B. E.; Call, D.; Cheng, S.; Hamelers, H. V. M.; Sleutels, T. J. A.; Jeremiasse, A. W.; Rozendal, R. A. Environ. Sci. Technol. 2008, 42 (23), 8630. doi: 10.1021/es801553z

(53) Wang, L. Y.; Chen, Y. G.; Huang, Q.; Feng, Y. Y.; Zhu, S. M.; Shen, S. B. J. Chem. Technol. Biotechnol. 2012, 87 (8), 1150. doi: $10.1002 /$ jctb.v87.8

(54) Zhang, Y. M.; Merrill, M. D.; Logan, B. E. Int. J. Hydrog. Energy 2010, 35 (21), 12020. doi: 10.1016/j.ijhydene. 2010.08 .064

(55) Selembo, P. A.; Merrill, M. D.; Logan, B. E. Int. J. Hydrog. Energy 2010, 35 (2), 428. doi: 10.1016/j.ijhydene.2009.11.014

(56) Hu, H.; Fan, Y.; Liu, H. Int. J. Hydrog. Energy 2010, 35 (8), 3227. doi: 10.1016/j.ijhydene.2010.01.131

(57) Tokash, J. C.; Logan, B. E. Int. J. Hydrog. Energy 2011, 36 (16), 9439. doi: 10.1016/j.ijhydene.2011.05.080

(58) Zhang, T.; Nie, H.; Bain, T. S.; Lu, H.; Cui, M.; SnoeyenbosWest, O. L.; Franks, A. E.; Nevin, K. P.; Russell, T. P.; Lovley, D. R. Energy Environ. Sci. 2013, 6 (1), 217. doi: 10.1039/ c2ee23350a

(59) Logan, B. E. ChemSusChem 2012, 5 (6), 988. doi: 10.1002/cssc. v5.6 